\title{
Hurdles in bacteriophage therapy: Deconstructing the parameters
}

\author{
Jessica Tsonos ${ }^{\mathrm{a}, \mathrm{b}, \mathrm{c}, \mathrm{d}}$, Dieter Vandenheuvel ${ }^{\mathrm{d}}$, Yves Briers ${ }^{\mathrm{d}}$, Henri De Greve ${ }^{\mathrm{b}, \mathrm{c}}$, \\ Jean-Pierre Hernalsteens ${ }^{\mathrm{a}}$, Rob Lavigne ${ }^{\mathrm{d}, *}$

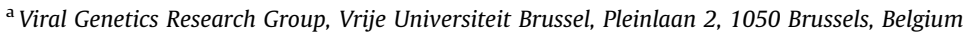 \\ ${ }^{\mathrm{b}}$ Structural and Molecular Microbiology, VIB, Vrije Universiteit Brussel, Pleinlaan 2, 1050 Brussels, Belgium \\ ${ }^{\mathrm{C}}$ Department Structural Biology, VIB, Vrije Universiteit Brussel, Pleinlaan 2, 1050 Brussels, Belgium \\ d Laboratory of Gene Technology, KU Leuven, Kasteelpark Arenberg 21, 3001 Leuven, Belgium
}

\section{A R T I C L E I N F O}

\section{Keywords:}

Phage therapy

Phage translocation

State of bacteria in vivo

Phage kinetics

\begin{abstract}
A B S T R A C T
Bacterial infections in animals impact our food production, leading to economic losses due to food rejection and the need for preventive and curative measures. Since the onset of the antibiotic era, the rise of antibiotic-resistant pathogens is causing scares in health care and food producing facilities worldwide. In the search of new therapeutics, re-evaluation of bacteriophage (phage) therapy, using naturally occurring bacterial viruses to tackle infections, is gaining interest. Many studies report about phage therapy success, showing the value and power of these natural viruses.

Although phages carry some interesting traits and their basic biology is now well understood, this review argues that phage therapy has not revealed all of its secrets and many parameters remain understudied, making the outcome of phage therapy highly variable depending on the disease incidence. These difficulties include poorly understood mechanisms of phage penetration and distribution throughout the body, the variable expression and accessibility of phage receptors on the bacterial host in in vivo conditions and the unusual (non-linear) phage pharmacokinetics. These parameters are not easily measured in realistic in vivo settings, but are nevertheless important hurdles to overcome the high variability of phage therapy trials. This critical approach is in accordance with Goethe's statement; "Difficulties increase the nearer we get to the goal". However, since the importance of the goal itself also rises, both difficulties and goal justify the need for additional in depth research in this domain.
\end{abstract}

(c) 2013 Elsevier B.V. All rights reserved.

\section{Introduction}

Bacterial infections are the cause of many human and animal health problems. The latter have a large impact on our food production, including meat, eggs and dairy. Product

\footnotetext{
* Corresponding author. Tel.: +32 163795 24; fax: +32 16321965.

E-mail addresses: jessica.tsonos@gmail.com (J. Tsonos), dieter.vandenheuvel@biw.kuleuven.be (D. Vandenheuvel), yves.briers@biw.kuleuven.be (Y. Briers), hdegreve@vub.ac.be (H. De Greve), jphernal@vub.ac.be (J.-P. Hernalsteens), rob.lavigne@kuleuven.be (R. Lavigne).
}

rejection, necessary to avoid human food-borne diseases, leads to huge economic losses for the farmers. The need to reduce bacterial infections in the food animal industry becomes clear in the Food and Agricultural Organization (FAO) statistical yearbook 2013, reporting an average annual meat consumption per capita of $80 \mathrm{~kg}$ in developed countries. In 2010, the worldwide meat production totaled to $3 \times 10^{8}$ tons (FAO, 2013). In 2011, the New York Times reported one of the largest meat recalls, being 36 million pounds of ground turkey, linked to a Salmonella outbreak where a strain resistant to many commonly prescribed antibiotics was involved (Neuman, August 4, 2011). 
The use of lytic bacterial viruses (bacteriophages), being the natural predators of bacteria, to treat bacterial infections and its efficient development in the veterinary sector may therefore have a significant economic impact, especially since antibiotic resistance becomes a major public health crisis. Antibiotic residues in food intended for human consumption and the compulsory withholding period to protect human from exposure to antibiotics would no longer be a concern if phage therapy is implemented at large scale. Indeed, phage therapy holds many intrinsic advantages. Bacteriophages are naturally occurring viruses that infect and lyse bacteria. They selfreplicate exponentially at the infection site and leave the commensal flora unaffected because of their narrow infection spectrum. Their possible autonomous transfer between animals could be useful in agricultural applications (Loc-Carrillo and Abedon, 2011). Because of their ubiquitous presence in nature, the isolation of phages active against specific bacterial strains should be simple.

The concept of phage therapy emerged quickly after the discovery of bacteriophages in the beginning of the 20th century by Twort and d'Herelle (Inal, 2003). After d'Herelle determined the safety of his phage preparation by oral and subcutaneous self-administration, he opened the door for the development of this new kind of therapy. With his commercial laboratory in Paris, d'Herelle was the first to introduce bacteriophages as therapeutic agents (Summers, 2001; Kutateladze and Adamia, 2008).

The history of phage therapy passed through four periods: enthusiasm, skepticism, abandonment and reappraisal. Early enthusiasm was generated by a set of encouraging results with the first ones being obtained by d'Herelle against "avian typhosis", a gastrointestinal disease, and bovine hemorrhagic septicemia (Summers, 2001). Several pharmaceutical firms started to sell phage preparations e.g., Eli Lilly, E.R Squibb and Sons, Antipol and The German Bacteriophage Society (Fischetti et al., 2006; Courchesne et al., 2009). The German and Soviet armies treated their soldiers during WWII against dysentery with phages (Hermoso et al., 2007). However, this excitement was short-lived and skepticism against phages as therapeutic agents took the lead. A lack of understanding of basic phage biology, quality control, absence of properly controlled studies and the associated unsuccessful therapeutic results contributed to this skepticism (Hanlon, 2007; Petty et al., 2007). Also, the discovery of penicillin saved thousands of Allied soldiers during WWII and its mass production was further developed in the post-war era to cure civilians and contributed to the downfall of phage therapy in Western medicine (Neushul, 1993). The Council of Pharmacy and Chemistry evaluated phage therapy in the late 1930s and acknowledged positive and negative results. Concern was expressed about the lack of standardization of phage preparations and the criteria for purity (Summers, 2001). In 1959, the World Health Organization came to the conclusion that there were no reasons to continue investigations with phages after the success of tetracycline against cholera (Barrow and Soothill, 1997; Barrow et al., 1998). Unfortunately, because of the widespread use (and misuse) of antibiotics, bacteria developed antibiotic resistance, in some cases to all clinically approved antibiotics. The potential of older methods had to be reexamined, explaining the renewed interest and reappraisal of phage therapy (Sulakvelidze, 2005).

Basic concepts we nowadays understand were completely unknown during the early period shortly after the discovery of phages. The two different replication cycles of phages (lytic \& lysogenic) have now been unraveled and the importance of the strictly lytic character of phages (limiting horizontal gene transfer between bacteria) in therapy is well established. In addition, the high-throughput sequencing technologies allow inexpensive sequencing, enabling us to assess the presence of genes coding for factors that increase or generate pathogenicity of the bacteria or for toxins (e.g., Shiga toxin, cholera toxin, botulinum neurotoxin) in the phage genome (Wagner and Waldor, 2002). Phage diversity and specificity was often underestimated and led to wrong phage choices for treatment in the past. Therapists were also not aware of the highly immunogenic character of the bacterial cell wall and outer membrane components found in crude phage lysates, which can lead to severe adverse reactions.

Even if our knowledge on bacteriophages is more extensive than ever before, the clinical outcome of phage therapy trials is still variable, indicating that there are still many parameters which are not yet understood or controlled. In the past, many attempts to improve the success of phage therapy have been based on the isolation of various phages from the environment and the identification of those that are the most effective in preventing mortality from a lethal infection (Levin and Bull, 2004). However, as will be shown in this review article, many more aspects are implicated than only the lytic capacity of phages and their simple administration to an infected organism.

In this review we outline the parameters which should be considered when analyzing the efficacy of whole phage therapy, to prevent the uncertainty and accidental outcome when going from an in vitro to an in vivo environment. We discuss the difficulties of phage penetration and its influence on therapeutic success. In addition, the importance of a detailed knowledge of the life style of the studied pathogen is highlighted, together with the too often neglected effect of the unusual phage pharmacokinetics. Where possible, available information concerning human phage therapy is mentioned, though human application remains subject to various (nonscientific) limitations (Verbeken et al., 2012). An outline of the review with the different topics is shown in Table 1.

\section{Bacteriophage administration and translocation in the body}

Depending on the site of infection and the method of administration, phages will need a high or low translocation capacity to reach the site of infection. At the industrial scale of intensive animal husbandry, phages must be administered to the food animals in a non-time-consuming and economically feasible manner. The most straightforward administration methods are either orally, by supplementing the feed or drinking water, or spraying 
Table 1

Selective reading table, giving an overview of treatment parameters and highlighted relevant reading.

\begin{tabular}{|c|c|c|c|c|}
\hline \multicolumn{4}{|l|}{ Key parameters for phage therapy } & \multirow[t]{2}{*}{ References } \\
\hline \multirow[t]{5}{*}{ Phage translocation } & Administration method & \multicolumn{2}{|c|}{ Target site } & \\
\hline & \multirow[t]{2}{*}{ Oral } & \multicolumn{2}{|l|}{ Gut } & $\begin{array}{l}\text { Oliveira et al. (2009) } \\
\text { Wagenaar et al. (2005) } \\
\text { Fiorentin et al. (2005) } \\
\text { Jamalludeen et al. (2009a) } \\
\text { Verthé et al. (2004) } \\
\text { McCallin et al. (2013) }\end{array}$ \\
\hline & & \multicolumn{2}{|c|}{ Bloodstream } & $\begin{array}{l}\text { Duerr et al. (2004) } \\
\text { Oliveira et al. (2009) }\end{array}$ \\
\hline & \multirow[t]{2}{*}{ Spray } & \multirow{2}{*}{\multicolumn{2}{|c|}{ Lungs }} & Huff et al. (2003) \\
\hline & & & & $\begin{array}{l}\text { Carmody et al. (2010) } \\
\text { Kvachadze et al. (2011) }\end{array}$ \\
\hline \multirow[t]{10}{*}{ Hindered host receptor binding } & & \multicolumn{2}{|l|}{ Caused by } & \\
\hline & Altered receptors & \multicolumn{2}{|l|}{ Phase variation } & $\begin{array}{l}\text { Bogomolnaya et al. (2008) } \\
\text { Kim and Ryu (2012) }\end{array}$ \\
\hline & & & Mandrell et al. (1990) \\
\hline & Shielded receptors & \multirow{2}{*}{\multicolumn{2}{|c|}{ Capsule production }} & Scholl et al. (2005) \\
\hline & & & & Snyder et al. (2004) \\
\hline & & \multirow{2}{*}{\multicolumn{2}{|c|}{$\begin{array}{l}\text { Colanic acid production } \\
\text { Intracellular location of pathogen }\end{array}$}} & Tuntufye et al. (2012) \\
\hline & Inaccessibility of receptors & & & Hsia et al. (2000) \\
\hline & & & & Capparelli et al. (2007) \\
\hline & & & & Broxmeyer et al. (2002) \\
\hline & & & & Hagens and Loessner (2007) \\
\hline \multicolumn{5}{|c|}{ Unusual pharmacokinetic properties } \\
\hline & \multirow[t]{4}{*}{ Subjected to density } & dent thresholds & Bacterial & Payne and Jansen (2001) \\
\hline & & & & Capparelli et al. (2007) \\
\hline & & & Phage & Payne et al., 2000 \\
\hline & & & & Abedon (2011) \\
\hline & \multicolumn{2}{|c|}{ Effect on the model of infection } & & Payne and Jansen (2003) \\
\hline
\end{tabular}

with subsequent inhalation. Topical and systemic delivery of phages is less straightforward in intensive animal husbandry systems because they demand an individualistic approach. Every infected animal would be treated in a personalized way, which is labor-intensive, timeconsuming and expensive. Therefore, these administration routes are limited to settings where these tailor-made treatments justify the increased costs, as in the treatment of valuable purebred animals and pets and in human medicine.

\subsection{Gastrointestinal delivery}

Oral administration would be the ideal way to treat gastrointestinal infections or colonization, e.g., caused by Campylobacter and Salmonella. These natural inhabitants of the gut of livestock are the most common and widely distributed causative agents of food-borne diseases because of meat contamination with intestinal content during processing (Carvalho et al., 2012). To reach the target site, phages have to pass the acidic environment of the stomach and the upper small intestine. However, phage have already shown a variable sensitivity against low pH in vitro (Jamalludeen et al., 2009b). Only one out of three lytic coliphages was detected in the duodenum above a certain dose ( $\geq 10^{7} \mathrm{PFU}$ ) after oral administration in chickens. This phage could not be detected after $24 \mathrm{~h}$ (Oliveira et al., 2009). In a study on the reduction of
Campylobacter jejuni colonization in young broilers, the single tested phage was able to remain "viable" while transiting the gastrointestinal tract, but with an important reduction in titer. With a daily phage administration $\left(10^{9}\right.$ $10^{10} \mathrm{PFU}$ ) over a 10 -days period in absence of a host, the detected plaque-forming units (PFU) per gram feces during this period varied between $10^{3}$ and $10^{5}$. Although a maximal $3 \log$ CFU reduction of $C$. jejuni could be obtained during phage administration, the bacterial number increased rapidly after the last phage dose and stabilized at a level of colonization only 10 times lower than the control group not receiving phage treatment (Wagenaar et al., 2005). Salmonella phages of an orally administered high titer $\left(3 \times 10^{11} \mathrm{PFU}\right)$ three-phage cocktail could be detected in the caecal content of treated birds at $\approx 10^{4} \mathrm{PFU} /$ $\mathrm{g}$ after the 15th day. However, phage identity was not confirmed, so uncertainty exists about the capacity of all three phages to reach the caecum. In the timeframe phages could be detected, the mean CFU in caecal content dropped about 200 times, while the CFU in untreated birds almost remained unchanged. When phages could no longer be detected, an important bacterial load of $2 \times 10^{6} \mathrm{CFU} / \mathrm{g}$ could still be measured (Fiorentin et al., 2005). Even though phage therapy in the above studies was not able to completely eradicate the presence of bacteria, the obtained results can be of importance. A quantitative risk assessment model suggested that a 2 log reduction of Campylobacter in chickens at time of slaughter would be sufficient 
to have a major impact on incidence of human disease (Rosenquist et al., 2003).

To reduce enterotoxigenic Escherichia coli (ETEC) colonization in pigs, six different phages were individually orally administered ( $\left.10^{10} \mathrm{PFU}\right)$. Even if some of the phages led to a significant positive weight change, and reduced the duration and severity of diarrhea, no phages could be detected with a theoretical detection limit being $10^{3} \mathrm{PFU} / \mathrm{g}$ feces. The mean percentage excretion of the challenge ETEC strain over total $E$. coli within the phage treated groups varied between $3 \%$ and $37 \%$, compared to $83 \%$ in the untreated control group. When taking a critical attitude, we have to admit that the given magnitudes do not represent a major decrease in CFUs, even when in some cases this could be enough to generate a physiological effect. With a different model of infection and prior oral administration of sodium bicarbonate to neutralize the stomach's $\mathrm{pH}$, phages of the three-phage cocktail $\left(10^{9} \mathrm{PFU}\right)$ could be detected in the feces for six days with extremely varying titers (between $10^{4}$ and $10^{11} \mathrm{PFU} / \mathrm{g}$ feces) (Jamalludeen et al., 2009a). Solutions to this decrease in phage titer may be a higher stomach $\mathrm{pH}$, which can be reached when oral phage administration is taking place shortly after feeding (Brüssow, 2005). In a study analyzing the stability of an Enterobacter aerogenes-specific phage under simulated gastrointestinal conditions, gastric $\mathrm{pH}$ has also been described as a major barrier when applying phages orally. At $\mathrm{pH} \mathrm{2,} \mathrm{the} \mathrm{phage} \mathrm{concentration} \mathrm{dropped}$ immediately below the detection limit $\left(1 \times 10^{1} \mathrm{PFU} / \mathrm{ml}\right)$. However in the reactors, supplemented with antacid $\left(\mathrm{NaHCO}_{3}\right)$, bile salts and pancreatin, there was no significant effect on the phage concentration during the $6 \mathrm{~h}$ of incubation (Verthé et al., 2004).

A first human small scale safety study relating to ingestion of a phage cocktail against E. coli and Proteus, was recently performed (McCallin et al., 2013). Fifteen healthy human subjects of different ages ingested an over-thecounter sold Russian phage cocktail. The participants were given a high, low and placebo dosage, with a one-week interval. Although the authors are well aware that delayed effects can occur which might therefore be misinterpreted as an effect of the dosage given, they have not encountered any adverse events associated with the phage dosages. Phage administration did not result in phages in the bloodstream and no change in the composition of the intestinal microbiota was observed. However, since the authors did not give any detailed information about presence of phages in feces, it cannot be assumed that the phages were stable throughout the gastrointestinal tract.

To cure systemic infections following oral administration, phages have to be able to translocate through the gastrointestinal barrier with relatively limited degradation to reach the peripheral blood and internal organs. In vivo phage display already showed the necessity of certain peptide sequences, expressed at the phage surface, to induce transcytosis across the intestinal barrier. It was demonstrated that the Enterobacteria phage M13 with unchanged structural proteins could not be recovered from the spleen of rats $2 \mathrm{~h}$ after gavage with $10^{12}$ PFU. M13 with a modified minor coat protein pIII, displaying a specific 7 amino acid sequence insert at the N-terminus, was able to translocate across the intestine and could be detected within intestinal mucosal epithelial and submucosal cells, while the unaltered M13 phage could only be observed in the intestinal chymus (Duerr et al., 2004). This reflects the possibility of modifying phages to enhance their translocation potential (should the use of modified phage be allowed). Apart from this record, there are some studies showing unmodified phages capable of passing through the intestinal wall of animals and humans to find their way to the bloodstream (Oliveira et al., 2009). It remains unclear whether phage enter the circulatory system via lymphatic vessels or through diffusion right into the bloodstream (Keller and Engley, 1958). Rectal administration seems also a way to deliver phages to the blood circulation. Penetration of phage to the blood circulation and urinary tract after oral administration was reported. Gastrointestinal administration for systemic phage delivery results in highly variable phage counts in the bloodstream, making the clinical importance of this administration route questionable (Weber-Dabrowska et al., 2000, 2003).

Phages must be able to pass by the immune system without being cleared. Miernikiewicz and colleagues show that neither $E$. coli phage T4 nor its major protein components affect the production of inflammation-related cytokines in mice, murine dendritic cell cultures or human blood samples (Miernikiewicz et al., 2013). Although the study has an in vivo aspect with the murine model, in vivo human data is lacking. Since this study is limited to the use of a single phage, it was stated that the human immune response to other phages has to be investigated, but the lack of stimulation of the immune system in this study is the first step, and supports the safety of intravenously administered phages.

\subsection{Pulmonary delivery}

Nebulizing phage suspensions can be used as an efficient way to inoculate all animals residing in the same animal housing. By spraying a phage preparation, fine droplets are inhaled. For respiratory tract infections, like avian pathogenic $E$. coli (APEC), often encountered in poultry farms, this administration method directly targets the phage to the infection site. Huff et al. (2003) were able to reduce the mortality of broiler chicks by spraying a twophage cocktail $2 \mathrm{~h}$ after APEC inoculation from 50\% for heat-killed phages, to $20 \%$ for active phages. The intramuscular (i.m.) administration of phages was, in contrast to spray administration, still effective when given after 24 and $48 \mathrm{~h}$. The researchers speculate that i.m. administration results in high systemic phage concentrations, which protect the birds against sepsis, whereas phage aerosols are not able to generate these protecting phage levels in blood (Huff et al., 2003). However, i.m. phage administration is not achievable on industrial production scale. It seems reasonable to assume that phages could penetrate the lungs, but confirmation for the presence of phage in the lungs after i.m. administration is lacking. The outcome of an intranasal or intraperitoneal phage treatment of a $24 \mathrm{~h}-$ old pulmonary Burkholderia cenocepacia infection was 
analyzed in mice (Carmody et al., 2010). The study showed that intraperitoneal administration was more effective than phage inhalation. The researchers did not specify the exact method of nebulization of the phage suspension, although a uniform delivery to the lower airways was claimed. At the end of their study, more phages could be recovered from the intranasal-treated mice, but also more viable bacteria (not significantly different from untreated mice) compared to the intraperitoneal treated mice. Intraperitoneal injection of phages led to the reduced presence of bacteria in the lungs, and was therefore the more efficient way of administration. The authors suggested that the intraperitoneally administered phages were more capable of reaching their host in the lung parenchyma. However, the drop in bacteria was only 1-2 logarithmic units, which may not be sufficient for an effective treatment in severely infected lungs. Nevertheless, the authors identified intraperitoneal phage delivery as a viable option for the treatment of lung infections (although this also will depend on economic feasibility).

In a more tailor-made manner, nebulization is used to treat pulmonary diseases in humans. To the best of our knowledge, the only human study describing inhalable phage therapy is a case study reporting the treatment of Staphylococcus aureus and Pseudomonas aeruginosa infection in a cystic fibrosis patient (Kvachadze et al., 2011). The existing phage cocktail Pyophage was supplemented with a phage specific against the infecting strain of $S$. aureus. No precise description of the nebulizing method of the phage suspension was provided, nor the administered antibiotic treatment, but the study showed improvement of the patient's condition and the absence of adverse effects of the phage treatment. The first clinical trials on larger groups of subjects remain to be published.

\section{The in vivo state of bacteria}

Assurance of phage receptor expression on the bacterial cell surface as well as its accessibility in in vivo conditions is of crucial importance to achieve success with phage therapy. Consequently, the bacterial state in vivo has a major impact on the potential of phage to infect and lyse them. Phage receptors, which are generally bacterial cell surface components, enable adsorption and have a key role in host specificity. Proteins and/or various lipopolysaccharide sections (Gram-negative bacteria), peptidoglycancontaining complexes (Gram-positive bacteria), capsular polysaccharides, pili and flagella can act as receptors (Lindberg, 1973). The primary requisite for bacteriophage infection is receptor recognition and successful attachment. This is dependent on the presence and accessibility of the receptor together with its localization and density on the cell surface (Rakhuba et al., 2010). Possible modifications in those parameters as a consequence of the adaptation of the pathogen to the in vivo environment will inevitably affect efficiency of phage infection. Pathogens entering the new environment of the host will react with their biochemical sensors to environmental stimuli and will respond by triggering a cascade of virulence factors which are critical for bacterial growth, survival and persistence during infection (Finlay and Falkow, 1989; Mahan et al., 2000). A coordinated expression of those virulence genes together with regulatory and metabolic genes is needed. Bacteria-phage interactions recurrently analyzed in vitro may be different in their destined in vivo environment, especially given the intraspecies variation in the response to such environments. The complex and dynamic milieu in animals cannot completely be reproduced in vitro. Although certain host environmental parameters can be mimicked in vitro to induce a subset of virulence genes, the full repertoire is only expressed in vivo (Angelichio and Camilli, 2002).

\subsection{Altered receptors}

Three genes (gtrABC1) have been described in Salmonella enterica serotype Typhimurium to cause 'form variation' upon exposure to eukaryotic cells (macrophages), by glucosylation of the 012 antigen generating the $12-2$ variant. Those genes are phase variably expressed and the 12-2 modification is only present on a small number of cells under bacterial culture conditions. It was shown that the deletion of one of those genes, with the associated incapacity of 012-antigen glucosylation, caused a decreased intestinal persistence when brought in competition with the wild type strain in Salmonellaresistant murine models of infection (Bogomolnaya et al. 2008). Phase variation is a gene regulation process which has been proposed to facilitate bacterial adaptation in a rapidly changing environment. Salmonella phage SPC35, for which it was shown that the 012-antigen of $S$. typhimurium assist the binding to its receptor BtuB during the initial phage adsorption step, is not able to infect Salmonella cells when the gtrABC1 genes are expressed (Kim and Ryu, 2012). Another report describes the physical presence of a component in vivo that induces enzymatic alternation of surface components. For example, the human pathogen Neisseria gonorrhoeae has an altered lipopolysaccharide in human serum, conferring serum resistance. The lipopolysaccharide is sialylated by a pathogen-encoded sialyltransferase in presence of cytidine monophospho-N-acetylneuraminic acid (CMP-NANA), which is present in blood. Because $N$. gonorrhoeae is not able to synthesize CMP-NANA and it is not present in the standard media, in vitro studies may have dealt with different lipopolysaccharide structures than those occurring in vivo (Mandrell et al., 1990).

\subsection{Shielded receptors}

Besides modification of the receptors, some bacteria shield their receptors against several phages through the production of extracellular polysaccharides that act as physical barrier. It has been demonstrated that the lipopolysaccharide-recognizing phage T7, is blocked from receptor adsorption by the exopolysaccharide $\mathrm{K} 1$ capsule in a K1-producing K12 E. coli strain (Scholl et al., 2005). The regulation of genes can be analyzed in vivo with the use of various technologies, including recombination-based in vivo expression technology (RIVET). Tuntufye et al. (2012) identified thereby $w c a J$ as a gene specifically expressed 
during systemic infection of APEC (avian pathogenic E. coli) in chickens. Its gene product is involved in the biosynthesis of an exopolysaccharide (colanic acid), forming a thick mucoid matrix on the cell surface (Tuntufye et al., 2012). The transcriptome of uropathogenic $E$. coli (UPEC) isolated from the urine of infected mice revealed a high upregulation of genes involved in capsular polysaccharide synthesis (Snyder et al., 2004). Capsule formation is often a virulence factor helping the pathogen to escape phagocytosis by the immune system (Finlay and Falkow, 1989).

\subsection{Inaccessible pathogen}

The intracellular form of some pathogens may also hinder traditional phage therapy, since phages must gain access to the intracellular location. This is especially the case for obligate intracellular parasites such as Chlamydia psittaci where the extracellular form is metabolically inert. Phages need for their replication metabolically active bacteria to avail host energy and biosynthesis. Transmission electron microscopy analysis demonstrated that cointernalization of the lytic phage $\varphi$ CPG1 occurred during eukaryotic incursion (HeLa cells), while they were attached to metabolically dormant elementary bodies of $C$. psittaci (Hsia et al., 2000). Infection of the pathogen in the eukaryotic vacuoles only took place after differentiation to an active bacterial form, with an abundant release of phage progeny after presumed phage-induced lysis. This observation of phage co-internalization has also been described during $S$. aureus infection of mouse peritoneal macrophages. The amount of intracellular bacteria could be reduced in vitro after treatment with phage-infected bacteria (i.e., phage adsorbed to $S$. aureus by preincubation). No reduction after treatment with phage alone could be observed (Capparelli et al., 2007). With the demonstrated need of a phage delivery vehicle to treat infections which have an intracellular form of life, a non-pathogenic Mycobacterium smegmatis strain has been proposed as "phage carrier" to reduce the intracellular numbers of pathogenic Mycobacterium avium. An in vitro 100-fold decrease of the pathogens present in infected macrophages was observed. The mechanism of phage TM4 delivery was observed by light-microscopy and showed the fusion of a vacuole containing $M$. smegmatis-TM4 with a vacuole containing M. avium (Broxmeyer et al., 2002). Because of the intracellular life style of Listeria monocytogenes during infection of the mammalian host, it has already been stated that classical phage therapy will be impossible in the treatment of the disease (Hagens and Loessner, 2007). However, Listeria phages are used as a preventive measure as food additive in high-risk foods, $c f$. Listex ${ }^{\mathrm{TM}}$ and ListShield $^{\mathrm{TM}}$ (Hagens and Loessner, 2007).

These findings highlight the need for a thorough understanding of the state and location of the targeted bacterium to adapt the phage treatment and angle of attack consequently.

\section{Specific pharmacokinetic properties}

A reason for diverse and unpredictable outcome when applying phages for therapeutic purposes has been argued by a lack of appreciation of the extent to which pharmacokinetics of phages differ from traditional molecule drugs. To enhance the successful outcome of phage therapy, a good understanding of the (non-linear) kinetics of bacteriophages as self-replicating pharmaceuticals is necessary, so that at least bacterial killing is theoretically likely when designing therapeutic protocols (Levin and Bull, 2004; Abedon, 2011). The best phage dosing, time of administration and choice of single/multiple administrations can be theoretically approached following computer simulations. Simple kinetic models, describing the rate at which the bacterial and phage concentrations evolve, rely on certain independently measurable biological parameters, the most important being adsorption rate, burst size, latent period (i.e. time between infection and lysis), phage decay rate and growth rate of bacteria (Payne and Jansen, 2003; Abedon, 2011). Some kinetic models incorporate parameters for bacterial resistance (mutation rate) and natural effects of the host, including the immune response (Payne and Jansen, 2001; Cairns et al., 2009). An estimation of some of these parameters is made following in vitro analysis. It must be emphasized that those phagebacteria interactions and the properties of phage and bacteria are likely to differ in vivo (Cairns et al., 2009), e.g., phage and bacterial titers seemed to be more stable in gastric juices with $\mathrm{pH}$ 2.0, compared to Lysogeny Broth with corresponding pH (Weld et al., 2004).

The applicability of simple generic growth models to a heterogeneous, less fluid environment of the digestive tract system of rats was tested. Those models were fitted in a crude approximation flow model to predict the bacterial and phage number evolution in the caecum. Those were compared to experimental data, where rats received orally a bacterial dose followed by a phage dose. For one of the tested phages (K1-5), the prediction of phage titer is in good agreement with the experimental data, even if the applied phage growth parameters were determined in vitro on exponentially growing bacteria. However, the bacteria were not eliminated in vivo in contrast to the simulation. Also, the second tested phage (T4) showed an in vivo growth deficiency with the predictions being far from the observed phage titers (Weld et al., 2004).

The models do not pretend to ensure successful therapeutic outcome of phage therapy trials but have to be considered as exploratory guidelines during phage choice, experimental design and result interpretation of in vivo tests.

\subsection{Existence of density-dependent thresholds}

Treatment outcome has been described by Payne and Jansen as being subjected to various density-dependent threshold effects (Payne and Jansen, 2001). The proliferation threshold, which is unique to phage therapy because of its self-replicating character, is of central importance in active phage therapy. A treatment is said to be active when it relies on phage replication in situ to achieve bacterial control (Curtright and Abedon, 2011). For active phage therapy, implying a net increase in phage numbers, a minimal bacterial concentration (proliferation threshold) is needed to ensure such a contact frequency that the rate 
of phage replication is higher than the rate at which they are lost. The proliferation threshold is dependent on phage and bacterial characteristics and highlights the importance of the time point of phage administration (Payne and Jansen, 2003). Ideally, active treatment should be given at the moment when bacterial densities reach a value where net phage increase can start, which is the proliferation onset time $\left(T_{p}\right)$. This is paradoxical when one compares it to antibiotics, where treatment has to be given as early as possible. Evidently, treatment shouldn't be administered too late, since mortality could in this case result from an irreversible, extreme toxin production or tissue damage by the pathogen (Bull et al., 2002). When an antibiotic is combined with a phage treatment, the effect of active therapy will, in accordance to those models, be reduced if $T_{p}$ is not attained because of antibiotic-induced bacterial killing (Payne and Jansen, 2003). This is of relevance in human phage therapy trials, where because of ethical reasons, phages are in almost all cases administered in combination with antibiotics. To administer the phage treatment at $T_{p}$, when active treatment wants to be achieved, is however even more complex when considering the different stages of infection in real circumstances at a population level, i.e. in cases where animals are kept in high volume housing.

Using an experimental approach, this bacterial density threshold where active treatment can take place, was found to be approximately $10^{4} \mathrm{CFU} / \mathrm{ml}$ in kidneys, spleen and heart of mice infected with $S$. aureus. In the bloodstream this threshold was not achieved and no net phage increase could be noticed (Capparelli et al., 2007). The famous experiment of Smith and Huggins (1982) achieved a more effective treatment using a single anti-K1 phage dose than multiple doses of antibiotic. This was linked to a strong active in vivo replication (Smith and Huggins, 1982). This implies a low and easily surpassed proliferation threshold (Payne and Jansen, 2001). The importance of good timing on the outcome of a successful treatment has thus been observed, but until now successful phage therapy is in many studies related to an early phage administration instead. This could be, for example, be illustrated by the subcutaneous infection of mice with Vibrio vulnificus, a human opportunistic pathogen, where optimum protection required phages to be administered (intraveneously) within $3 \mathrm{~h}$ of bacterial inoculation (Cerveny et al., 2002). Furthermore, immediate treatment could be necessary in those cases where a local infection evolves to a general infection. Treatment of poultry suffering from experimentally induced severe E. coli respiratory infection with an aerosol phage spray showed a decrease in mortality when phage was administered immediately after infection, while only minor treatment efficacy was obtained when phage were given after 24 or $48 \mathrm{~h}$ (Huff et al., 2003). A reduction in mortality in a mouse model infected by Salmonella could only be obtained for a certain oral treatment schedule, with the earliest (hours post infection) the most efficient, while other treatments (days post infection) only delayed the death of the mice (Bardina et al., 2012).

Besides bacterial density, the administered phage quantity is also highly important for the outcome of phage therapy. For phage therapy likely to be effective, the dose of phage present at the infection site must be higher than the inundation threshold, the concentration of free phage inducing a reduction in bacterial amounts (Payne et al., 2000; Payne and Jansen, 2001). This threshold can be reached immediately or can be delayed in time because of in situ phage replication (if conditions enable active therapy). As deduced from mathematical models, it has been described as being the ratio of the rate of bacterial replication over the rate of phage adsorption (Abedon, 2011). As stated by Abedon, this is a metric that is difficult to determine in situ and can only avoid that chosen phage densities are far too low to achieve desired levels of phage therapy efficacy (Abedon, 2011). This can easily be illustrated by many studies, one of them being the recovery of 5/5 mice after intravenous treatment with $10^{9}$ PFU Staphylococcus phages, compared to $100 \%$ deaths with a dose of $10^{7}$ PFU (Capparelli et al., 2007).

If conditions do not enable active therapy, a continuous delivery of concentrated phages will be needed. Passive treatment supplies sufficiently high phage densities, without relying on in situ phage replication. Under these circumstances the larger and the earlier the dose of phage the better (equivalent to conventional drugs). However, for certain pathologies, phage will need the capacity to translocate to reach the bacterial reservoir in sufficiently high numbers to reduce the rate of replication of infecting bacteria, which can be difficult to achieve (see above).

\subsection{Importance of the model of infection}

When designing an animal experiment attention must be paid to the natural host time scale. This is the time point at which an untreated animal would recover or die. Active therapy can only be feasible if the proliferation onset time $\left(T_{p}\right)$ is smaller than the natural host time scale. If the pathogen is too virulent and the animal dies at low bacterial load, even before $T_{p}$ could be reached, effective active therapy will theoretically be impossible. In this case only passive therapy can be applied. From the other side, the efficacy of an active treatment can also not be assessed if the animal recovers before $T_{p}$ is reached (Payne and Jansen, 2003). Active therapy will fail when tested on models of infection with a too low bacterial infection dose or rapid bacterial dissemination in the animal's body, inducing too low bacterial densities accompanied by a bad timing of phage administration (and too low titer). These principles should be kept in mind when the bacterial strain and the bacterial inoculum size are chosen to assess the efficacy of a phage (cocktail) in experimentally infected animals.

Successful in vivo experiments in experimental animal models may, however, not guarantee success of phage therapy under realistic conditions, where bacterial densities cannot be monitored in real time, especially given the natural variation of virulence among strains of the same species.

\section{Conclusion}

This review on natural bacteriophage therapy was made with the objective to highlight some points which 
are not yet properly understood. Some factors are crucial for the outcome of phage therapy and should not be underestimated. Hurdles caused by the intrinsic nature of the phage need to be overcome. We are still at a stage where a strong variability exists on the outcome of whole phage therapy. The view on its success may be biased from the fact that most negative results remain unpublished. The most, and maybe only, critical view on phage therapy has been described in articles reporting the peculiar kinetics of these self-replicating pharmaceuticals. Payne and co-workers commented the studies on bacteriophage therapy as having a history of being rather hit-or-miss. Phages that actively replicate and lyse bacteria in vitro do not always do so in vivo explaining the high variability of therapeutic outcome of whole phage administration after infection (Payne et al., 2000).

Whole phage therapy certainly has potential as a treatment in specific areas. Its efficiency has been reported in several cases and several companies are undertaking the commercialization of bacteriophages. Intralytix Inc. produces besides phage preparations for food application (ListShield $^{\mathrm{TM}}$ against Listeriosis, EcoShield ${ }^{\mathrm{TM}}$ against E. coli O157:H7, SalmoFresh ${ }^{\mathrm{TM}}$ against salmonellosis), bacteriophage-products for veterinary applications (PLSV-1 $1^{\mathrm{TM}}$ against Salmonella and INT-401 ${ }^{\mathrm{TM}}$ against Clostridium perfringens in poultry) and two human therapeutic products which are in various stages of development. Micreos has commercialized LISTEX ${ }^{\mathrm{TM}}$ against L. monocytogenes as a food processing-aid and several trials relating to animal health are ongoing. Ampliphi is analyzing phage mixtures in the preclinical stage for human and animal therapy.

The hurdle of translocation is not present in case of topical phage delivery and its applicability is therefore less complicated. Phages have shown to be promising therapeutics in the treatment of burn wounds. Often, antibiotics are not capable of reaching burn wound beds in sufficient high concentrations to clear bacterial infections. PhagoBioDerm, a biodegradable polymeric compress impregnated with bacteriophages, was licensed for sale in the Republic of Georgia in 1999, and a complete recovery or improvement of clinical symptoms in almost $95 \%$ of the patients was reported (Markoishvili et al., 2002). However, since 1999 there has been no widespread distribution of the product. Marza and colleagues report a successful case study of phage treatment applied on burn wounds colonized with P. aeruginosa (Marza et al., 2006). Ten pet dogs with difficult to clear, antibiotic-resistant otitis caused by $P$. aeruginosa were treated with a six-phage cocktail (Hawkins et al., 2010). Two days after phage administration, bacterial counts fell in all cases, whereas phage counts were higher than the initial administered dose.

The more translocation is necessary for phages to reach their infection site, the more hurdles they will need to overcome. Depending on their characteristics, variable numbers of phages will arrive at the end of this obstacle course and will or will not reach the target infection site. For this, a good phage selection and corresponding titer will be important to bring at the infection site the minimal amount of phage necessary for active or passive therapy to be effective. To achieve successful active phage therapy, one should take into account many complex parameters which are hard to determine for in vivo situations. For this reason passive therapy seems to be more likely to be a good first option of treatment. Especially in this last case, if highly concentrated phages are required for treatment, one should evaluate the technological capacity to achieve such high concentrations and associated costs for production. Intramuscular phage administration has already shown to deliver higher phage levels in the blood, which in case of systemic infections will be more able to diminish bacterial load (Huff et al., 2003). Such treatments are on industrial production scale less easily feasible or even not conceivable, e.g. in poultry farms. Limitations following restrictions in time and cost in the food animal production sector will need adapted treatments.

Because of the difficulty to predict the in vivo efficacy based on in vitro results, as stated above, Henry and colleagues proposed a method to assess the therapeutic potential of phages based on a real-time imaging system in a mice lung infection model. Two of the nine individually tested phages, with in vitro activity, did not show the expected in vivo therapeutic capacity to rescue infected animals in similar conditions. One of them had therapeutic effect after increasing the phage dose ( $c f$. kinetics), the other one when a different bacterial infection strain was used ( $c f$. bacterial state in vivo). By paralleling the evolution in bacterial bioluminescence with the proportion surviving bacteria, a model was developed whereby bacteriophages could be discriminated based on their in vivo efficacy after $8 \mathrm{~h}$. The development of such models would contribute in the rapid screening of therapeutic efficient phages in vivo (Henry et al., 2013).

Further analysis of these and other domain-specific parameters, will allow to determine the limitations and the applicability of phage therapy.

\section{Conflict of interest statement}

We declare no competing interests.

\section{References}

Abedon, S., 2011. Phage therapy pharmacology: calculating phage dosing. Adv. Appl. Microbiol. 77, 1-40.

Angelichio, M.J., Camilli, A., 2002. In vivo expression technology. Infect. Immun. 70, 6518-6523.

Bardina, C., Spricigo, D.A., Cortés, P., Llagostera, M., 2012. Significance of the bacteriophage treatment schedule in reducing Salmonella colonization of poultry. Appl. Environ. Microbiol. 78, 6600-6607.

Barrow, P., Lovell, M., Berchieri, A., 1998. Use of lytic bacteriophage for control of experimental Escherichia coli septicemia and meningitis in chickens and calves. Clin. Diagn. Lab. Immunol. 5, 294-298.

Barrow, P.A., Soothill, J.S., 1997. Bacteriophage therapy and prophylaxis: rediscovery and renewed assessment of potential. Trends Microbiol. $5,268-271$.

Bogomolnaya, L.M., Santiviago, C.A., Yang, H.J., Baumler, A.J., AndrewsPolymenis, H.L., 2008. 'Form variation' of the 012 antigen is critical for persistence of Salmonella typhimurium in the murine intestine. Mol. Microbiol. 70, 1105-1119.

Broxmeyer, L., Sosnowska, D., Miltner, E., Chacón, O., Wagner, D., McGarvey, J., Barletta, R.G., Bermudez, L.E., 2002. Killing of Mycobacterium avium and Mycobacterium tuberculosis by a mycobacteriophage delivered by a nonvirulent mycobacterium: a model for phage therapy of intracellular bacterial pathogens. J. Infect. Dis. 186, 1155-1160. 
Brüssow, H., 2005. Phage therapy: the Escherichia coli experience. Microbiology 151, 2133-2140.

Bull, J.J., Levin, B.R., DeRouin, T., Walker, N., Bloch, C.A., 2002. Dynamics of success and failure in phage and antibiotic therapy in experimental infections. BMC Microbiol. 2, 35.

Cairns, B.J., Timms, A.R., Jansen, V.A., Connerton, I.F., Payne, R.J., 2009. Quantitative models of in vitro bacteriophage-host dynamics and their application to phage therapy. PLoS Pathog. 5, e1000253.

Capparelli, R., Parlato, M., Borriello, G., Salvatore, P., Iannelli, D., 2007. Experimental phage therapy against Staphylococcus aureus in mice. Antimicrob. Agents Chemother. 51, 2765-2773.

Carmody, L.A., Gill, J.J., Summer, E.J., Sajjan, U.S., Gonzalez, C.F., Young, R.F., LiPuma, J.J., 2010. Efficacy of bacteriophage therapy in a model of Burkholderia cenocepacia pulmonary infection. J. Infect. Dis. 201, 264271.

Carvalho, C., Santos, S., Kropinski, A., Ferreira, E., Azeredo, J., 2012. Phages as therapeutic tools to control major foodborne pathogens: Campylobacter and Salmonella. In: Kurtboke, D.I. (Ed.), Bacteriophages InTech, Rijeka, pp. 179-214.

Cerveny, K.E., DePaola, A., Duckworth, D.H., Gulig, P.A., 2002. Phage therapy of local and systemic disease caused by Vibrio vulnificus in iron-dextran-treated mice. Infect. Immun. 70, 6251-6262.

Courchesne, N.M., Parisien, A., Lan, C.Q., 2009. Production and application of bacteriophage and bacteriophage-encoded lysins. Recent Pat. Biotechnol. 3, 37-45.

Curtright, A.J., Abedon, S.T., 2011. Phage therapy: emergent property pharmacology. J. Bioanal. Biomed. S6, 002.

Duerr, D.M., White, S.J., Schluesener, H.J., 2004. Identification of peptide sequences that induce the transport of phage across the gastrointestinal mucosal barrier. J. Virol. Methods 116, 177-180.

FAO, 2013. Food and Agricultural Organization of the United Nations. World Food and Agriculture, Rome.

Finlay, B.B., Falkow, S., 1989. Common themes in microbial pathogenicity. Microbiol. Rev. 53, 210-230.

Fiorentin, L., Vieira, N.D., Barioni, W., 2005. Oral treatment with bacteriophages reduces the concentration of Salmonella Enteritidis PT4 in caecal contents of broilers. Avian Pathol. 34, 258-263.

Fischetti, V.A., Nelson, D., Schuch, R., 2006. Reinventing phage therapy: are the parts greater than the sum? Nat. Biotechnol. 24, 1508-1511.

Hagens, S., Loessner, M.J., 2007. Listeria monocytogenes: Pathogenesis and Host Response. Springer, US

Hanlon, G.W., 2007. Bacteriophages: an appraisal of their role in the treatment of bacterial infections. Int. J. Antimicrob. Agents 30, 118128

Hawkins, C., Harper, D., Burch, D., Anggård, E., Soothill, J., 2010. Topical treatment of Pseudomonas aeruginosa otitis of dogs with a bacteriophage mixture: a before/after clinical trial. Vet. Microbiol. 146, 309313

Henry, M., Lavigne, R., Debarbieux, L., 2013. Predicting in vivo efficacy to guide the choice of therapeutic bacteriophages to treat pulmonary infections. Antimicrob. Agents Chemother. 57, 5961-5968.

Hermoso, J.A., García, J.L., García, P., 2007. Taking aim on bacterial pathogens: from phage therapy to enzybiotics. Curr. Opin. Microbiol. 10, 461-472.

Hsia, R., Ohayon, H., Gounon, P., Dautry-Varsat, A., Bavoil, P.M., 2000. Phage infection of the obligate intracellular bacterium. Chlamydia psittaci strain guinea pig inclusion conjunctivitis. Microbes Infect. 2, $761-772$.

Huff, W.E., Huff, G.R., Rath, N.C., Balog, J.M., Donoghue, A.M., 2003. Evaluation of aerosol spray and intramuscular injection of bacteriophage to treat an Escherichia coli respiratory infection. Poult. Sci. 82, 1108-1112.

Inal, J.M., 2003. Phage therapy: a reappraisal of bacteriophages as antibiotics. Arch. Immunol. Ther. Exp. (Warsz) 51, 237-244.

Jamalludeen, N., Johnson, R.P., Shewen, P.E., Gyles, C.L., 2009a. Evaluation of bacteriophages for prevention and treatment of diarrhea due to experimental enterotoxigenic Escherichia coli 0149 infection of pigs. Vet. Microbiol. 136, 135-141.

Jamalludeen, N., She, Y.M., Lingohr, E.J., Griffiths, M., 2009b. Isolation and characterization of virulent bacteriophages against Escherichia coli serogroups 01, O2, and 078. Poult. Sci. 88, 1694-1702.

Keller, R., Engley Jr., F.B., 1958. Fate of bacteriophage particles introduced into mice by various routes. Proc. Soc. Exp. Biol. Med. 98, 577-580.

Kim, M., Ryu, S., 2012. Spontaneous and transient defence against bacteriophage by phase-variable glucosylation of O-antigen in Salmonella enterica serovar Typhimurium. Mol. Microbiol. 86, 411-425.

Kutateladze, M., Adamia, R., 2008. Phage therapy experience at the Eliava Institute. Med. Mal. Infect. 38, 426-430.

Kvachadze, L., Balarjishvili, N., Meskhi, T., Tevdoradze, E., Skhirtladze, N., Pataridze, T., Adamia, R., Topuria, T., Kutter, E., Rohde, C., Kutateladze,
M., 2011. Evaluation of lytic activity of staphylococcal bacteriophage Sb-1 against freshly isolated clinical pathogens. Microb. Biotechnol. 4, 643-650.

Levin, B.R., Bull, J.J., 2004. Population and evolutionary dynamics of phage therapy. Nat. Rev. Microbiol. 2, 166-173.

Lindberg, A.A., 1973. Bacteriophage receptors. Annu. Rev. Microbiol. 27, 205-241.

Loc-Carrillo, C., Abedon, S.T., 2011. Pros and cons of phage therapy. Bacteriophage 1, 111-114.

Mahan, M.J., Heithoff, D.M., Sinsheimer, R.L., Low, D.A., 2000. Assessment of bacterial pathogenesis by analysis of gene expression in the host. Annu. Rev. Genet. 34, 139-164.

Mandrell, R.E., Lesse, A.J., Sugai, J.V., Shero, M., Griffiss, J.M., Cole, J.A. Parsons, N.J., Smith, H., Morse, S.A., Apicella, M.A., 1990. In vitro and in vivo modification of Neisseria gonorrhoeae lipooligosaccharide epitope structure by sialylation. J. Exp. Med. 171, 1649-1664.

Markoishvili, K., Tsitlanadze, G., Katsarava, R., Morris, J.G., Sulakvelidze, A., 2002. A novel sustained-release matrix based on biodegradable poly(ester amide)s and impregnated with bacteriophages and an antibiotic shows promise in management of infected venous stasis ulcers and other poorly healing wounds. Int. J. Dermatol. 41, 453-458.

Marza, J.A., Soothill, J.S., Boydell, P., Collyns, T.A., 2006. Multiplication of therapeutically administered bacteriophages in Pseudomonas aeruginosa infected patients. Burns 32, 644-646.

McCallin, S., Alam Sarker, S., Barretto, C., Sultana, S., Berger, B., Huq, S., Krause, L., Bibiloni, R., Schmitt, B., Reuteler, G., Brussow, H., 2013. Safety analysis of a Russian phage cocktail: from metagenomic analysis to oral application in healthy human subjects. Virology 443 , 187-196.

Miernikiewicz, P., Dabrowska, K., Piotrowicz, A., Owczarek, B., WojasTurek, J., Kicielinska, J., Rossowska, J., Pajtasz-Piasecka, E., Hodyra, K., Macegoniuk, K., Rzewucka, K., Kopciuch, A., Majka, T., Letarov, A., Kulikov, E., Maciejewski, H., Gorski, A., 2013. T4 phage and its head surface proteins do not stimulate inflammatory mediator production. PLOS ONE 8, e71036.

Neuman, W., 2011. Cargill Issues Meat Recall after Turkey Tied to Illness In The New York Times, New York (August 4).

Neushul, P., 1993. Science, government, and the mass production of penicillin. J. Hist. Med. Allied Sci. 48, 371-395.

Oliveira, A., Sereno, R., Nicolau, A., Azeredo, J., 2009. The influence of the mode of administration in the dissemination of three coliphages in chickens. Poult. Sci. 88, 728-733.

Payne, R.J., Jansen, V.A., 2001. Understanding bacteriophage therapy as a density-dependent kinetic process. J. Theor. Biol. 208, 37-48.

Payne, R.J., Jansen, V.A., 2003. Pharmacokinetic principles of bacteriophage therapy. Clin. Pharmacokinet. 42, 315-325.

Payne, R.J., Phil, D., Jansen, V.A., 2000. Phage therapy: the peculiar kinetics of self-replicating pharmaceuticals. Clin. Pharmacol. Ther. 68, 225230.

Petty, N.K., Evans, T.J., Fineran, P.C., Salmond, G.P., 2007. Biotechnological exploitation of bacteriophage research. Trends Biotechnol. 25, 7-15.

Rakhuba, D.V., Kolomiets, E.I., Dey, E.S., Novik, G.I., 2010. Bacteriophage receptors, mechanisms of phage adsorption and penetration into host cell. Pol. J. Microbiol. 59, 145-155.

Rosenquist, H., Nielsen, N.L., Sommer, H.M., Nørrung, B., Christensen, B.B., 2003. Quantitative risk assessment of human campylobacteriosis associated with thermophilic Campylobacter species in chickens. Int. J. Food Microbiol. 83, 87-103.

Scholl, D., Adhya, S., Merril, C., 2005. Escherichia coli K1's capsule is a barrier to bacteriophage T7. Appl. Environ. Microbiol. 71, 4872-4874.

Smith, H.W., Huggins, M.B., 1982. Successful treatment of experimental Escherichia coli infections in mice using phage: its general superiority over antibiotics. J. Gen. Microbiol. 128, 307-318.

Snyder, J.A., Haugen, B.J., Buckles, E.L., Lockatell, C.V., Johnson, D.E., Donnenberg, M.S., Welch, R.A., Mobley, H.L., 2004. Transcriptome of uropathogenic Escherichia coli during urinary tract infection. Infect. Immun. 72, 6373-6381.

Sulakvelidze, A., 2005. Phage therapy: an attractive option for dealing with antibiotic-resistant bacterial infections. Drug Discov. Today 10 807-809.

Summers, W.C., 2001. Bacteriophage therapy. Annu. Rev. Microbiol. 55, 437-451

Tuntufye, H.N., Lebeer, S., Gwakisa, P.S., Goddeeris, B.M., 2012. Identification of Avian pathogenic Escherichia coli genes that are induced in vivo during infection in chickens. Appl. Environ. Microbiol. 78 3343-3351.

Verbeken, G., Pirnay, J.P., De Vos, D., Jennes, S., Zizi, M., Lavigne, R. Casteels, M., Huys, I., 2012. Optimizing the European regulatory framework for sustainable bacteriophage therapy in human medicine. Arch. Immunol. Ther. Exp. (Warsz) 60, 161-172. 
Verthé, K., Possemiers, S., Boon, N., Vaneechoutte, M., Verstraete, W., 2004. Stability and activity of an Enterobacter aerogenes-specific bacteriophage under simulated gastro-intestinal conditions. Appl. Microbiol. Biotechnol. 65, 465-472.

Wagenaar, J.A., Van Bergen, M.A., Mueller, M.A., Wassenaar, T.M., Carlton, R.M., 2005. Phage therapy reduces Campylobacter jejuni colonization in broilers. Vet. Microbiol. 109, 275-283.

Wagner, P.L., Waldor, M.K., 2002. Bacteriophage control of bacterial virulence. Infect. Immun. 70, 3985-3993.
Weber-Dabrowska, B., Mulczyk, M., Gorski, A., 2000. Bacteriophage therapy of bacterial infections: an update of our institute's experience. Arch. Immunol. Ther. Exp. (Warsz) 48, 547-551.

Weber-Dabrowska, B., Mulczyk, M., Gorski, A., 2003. Bacteriophages as an efficient therapy for antibiotic-resistant septicemia in man. Transplant. Proc. 35, 1385-1386.

Weld, R.J., Butts, C., Heinemann, J.A., 2004. Models of phage growth and their applicability to phage therapy. J. Theor. Biol. 227, 1-11. 